\title{
Wave localization in one-dimensional random structures composed of single-negative metamaterials
}

\author{
P. Han, ${ }^{1,2}$ C. T. Chan, ${ }^{1}$ and Z. Q. Zhang ${ }^{1}$ \\ ${ }^{1}$ Department of Physics, The Hong Kong University of Science and Technology, Clear Water Bay, Kowloon, Hongkong, China \\ ${ }^{2}$ School of Physics and Telecommunication Engineering, South China Normal University, Guangzhou 510631, China
}

(Received 5 September 2007; revised manuscript received 19 November 2007; published 18 March 2008)

\begin{abstract}
By using the transfer-matrix method, we study the Anderson localization behavior in one-dimensional random systems composed of two different single-negative (SNG) metamaterials, in which either the permittivity or the permeability is negative. Both nondispersive and dispersive models have been adopted in this study. We find that when both the averaged permittivity and the averaged permeability are negative, or effectively a negative refractive index, the localization behavior in the long-wavelength limit is found to be similar to that of the traditional random systems made of double-positive (DP) materials, i.e., positive permittivity and positive permeability, and can be described by the standard localization theory developed for DP materials, although the wave transport mechanism of such systems is very different from that of DP materials. In the case of a dispersive model, a different localization behavior has been found inside a gap created around a particular frequency at which the sum of the impedances of the two SNG metamaterials vanishes. For example, the frequency dependence of the localization length can exhibit a sharp peak inside the gap, and the localization length is found to be smaller than the decay length of the corresponding periodic structure. The latter is opposite to the well-known localization behavior found in DP materials, where the localization length is, in general, larger than the decay length. Various wave propagation properties associated with this gap have been obtained. Some analytical results based on transfer matrices and long-wavelength limit description have been used to explain the simulation results.
\end{abstract}

DOI: $10.1103 /$ PhysRevB.77.115332

PACS number(s): 42.25.Dd, 41.20.Jb, 78.67.Pt, 78.20.Ci

\section{INTRODUCTION}

Recently, a new type of artificial composite, so-called metamaterials, has attracted a great deal of attention. The most interesting characteristic of these metamaterials is their negative permittivity $\varepsilon$ and/or negative permeability $\mu .{ }^{1}$ In practice, the negative $\varepsilon$ and negative $\mu$ are realized by using subwavelength resonators such as split rings and small metallic wires, ${ }^{2}$ respectively. One can construct metamaterials with both negative $\varepsilon$ and $\mu$, so-called double-negative (DNG) metamaterials or left-handed materials. At the same time, one can also construct materials with only one of $\varepsilon$ and $\mu$ negative, i.e., $\varepsilon<0$ and $\mu>0$, or $\varepsilon>0$ and $\mu<0$, socalled single-negative (SNG) metamaterials.

It has been reported that structures containing these metamaterials show some unusual electromagnetic properties. For example, a slab of DNG materials can be used as a superlens; ${ }^{3}$ a periodical arrangement of layers made of ordinary and DNG metamaterials can display zero- $\bar{n}$ band gap, ${ }^{4}$ while a periodical arrangement of SNG metamaterials shows a SNG stop band; ;,6 one-dimensional (1D) periodical structures containing layers of DNG materials may exhibit a three-dimensional band gap, ${ }^{7}$ and so on.

Most of the previous works on metamaterials focused on certain unusual properties of wave propagation in a periodical structure. A natural question to ask is, how would these properties be altered if randomness is introduced into the structures? This problem has been extensively studied for traditional random systems made of double-positive (DP) materials with both $\varepsilon>0$ and $\mu>0$. For DP materials, it is now well accepted that all states are localized in one dimension when any amount of randomness is introduced into the system. ${ }^{8,9}$ Since periodical systems containing metamaterials have been demonstrated to possess many unusual properties, nontrivial localization properties can also be expected when randomness is introduced into such systems. Recently, wave propagation in 1D random systems made of alternating layers of DP and DNG metamaterials has been studied and some novel localization length behaviors have been found. ${ }^{10,11}$

In this work, by using the transfer-matrix method, we study wave localization behavior in one-dimensional random systems made of two kinds of SNG metamaterials. Both nondispersive and dispersive models are adopted in this study. Our focus is in the long-wavelength limit. Our results show that the localization behavior in a propagating band, which effectively possesses a negative refractive index with both the averaged permittivity and averaged permeability being negative, is in general similar to that found in the random systems made of DP materials and can be described by the standard localization theory developed for DP materials. However, the wave transport mechanism of such systems is very different from that of DP materials. In the case of a dispersive model, the random SNG systems do display some unique features which are in sharp contrast to the case of traditional random DP systems. This occurs when the sum of the impedances of the two different SNG metamaterials vanishes. Around this frequency, a gap can be created. Within this gap, the localization length is found to be smaller than the decay length of the corresponding periodical system. This is opposite to the well-known localization behavior found in DP materials, where the localization length is, in general, larger than the decay length. The frequency dependence of the localization length can also exhibit a sharp peak 
inside the gap. We explain these anomalous phenomena as well as other wave propagation properties associated with this gap by using transfer matrices together with a longwavelength limit description.

\section{MODEL DESCRIPTION}

We consider the propagation of electromagnetic (EM) waves through a random one-dimensional sample consisting of equal numbers of two kinds of SNG layers: $A$ with $\varepsilon_{A}$ $<0$ and $\mu_{A}>0$, and $B$ with $\varepsilon_{B}>0$ and $\mu_{B}<0$ embedded in air. The layer thicknesses are fixed as $d_{A}$ and $d_{B}$, respectively. The random structure is constructed by stacking these two kinds of layers in random order. Since we do not allow layers to overlap, the sample thickness remains the same for all different random configurations, i.e., $L=N\left(d_{A}+d_{B}\right)$, where $N$ is the number of $A$ and $B$ layers. We assume that the EM wave is incident normally on one surface of the sample. We calculate the transmission coefficient on the other surface of the sample. Since only one of $\varepsilon$ and $\mu$ in such SNG metamaterials is negative, the refractive index in each layer is purely imaginary and, therefore, the field inside these SNG layers is evanescent. When the system is periodic with a pair of $A$ and $B$ layers as the unit cell, band structures with both propagating and forbidden bands can appear in such system. Its dispersion relation can be obtained from the Bloch-Floquet theorem, ${ }^{12}$ i.e.,

$$
\begin{aligned}
\cos K \Lambda= & \cos \left(k_{A} d_{A}\right) \cos \left(k_{B} d_{B}\right)-\frac{1}{2}\left(\frac{q_{B}}{q_{A}}\right. \\
& \left.+\frac{q_{A}}{q_{B}}\right) \sin \left(k_{A} d_{A}\right) \sin \left(k_{B} d_{B}\right),
\end{aligned}
$$

where $K$ is the Bloch wave vector and $\Lambda=d_{A}+d_{B}$ is the period. $q_{i}=\sqrt{\mu_{i}} / \sqrt{\varepsilon_{i}}$ and $k_{i}=k \sqrt{\varepsilon_{i}} \sqrt{\mu_{i}}(i=A, B)$, and $k$ is the wave vector in vacuum. It should be noted that both the wave vector $k_{i}$ and the impedance $q_{i}$ are purely imaginary due to the negativity of one of $\varepsilon_{i}$ and $\mu_{i}$. Therefore, Eq. (1) becomes ${ }^{5,6}$

$$
\begin{aligned}
\cos K \Lambda= & \cosh \left(\left|k_{A}\right| d_{A}\right) \cosh \left(\left|k_{B}\right| d_{B}\right)-\frac{1}{2}\left(\frac{\left|q_{B}\right|}{\left|q_{A}\right|}\right. \\
& \left.+\frac{\left|q_{A}\right|}{\left|q_{B}\right|}\right) \sinh \left(\left|k_{A}\right| d_{A}\right) \sinh \left(\left|k_{B}\right| d_{B}\right) .
\end{aligned}
$$

Since we take the absolute values of $k_{i}$ and $q_{i}$ in Eq. (2), the hyperbolic functions of cosh and sinh are used. The condition that a frequency is in a pass band or a gap depends on whether the value on the right-hand side of Eq. (2) is between -1 and 1 or not. When the value falls outside this range, $K$ becomes complex, which implies that the frequency is inside a gap.

The formation of band structures in such system is different from that in a system made of DP materials, which is known as the Bragg scattering. Since the wave vector in each of the SNG layer is purely imaginary, it is important to understand the wave transport mechanism inside a pass band. We first consider the transmission of EM waves through a

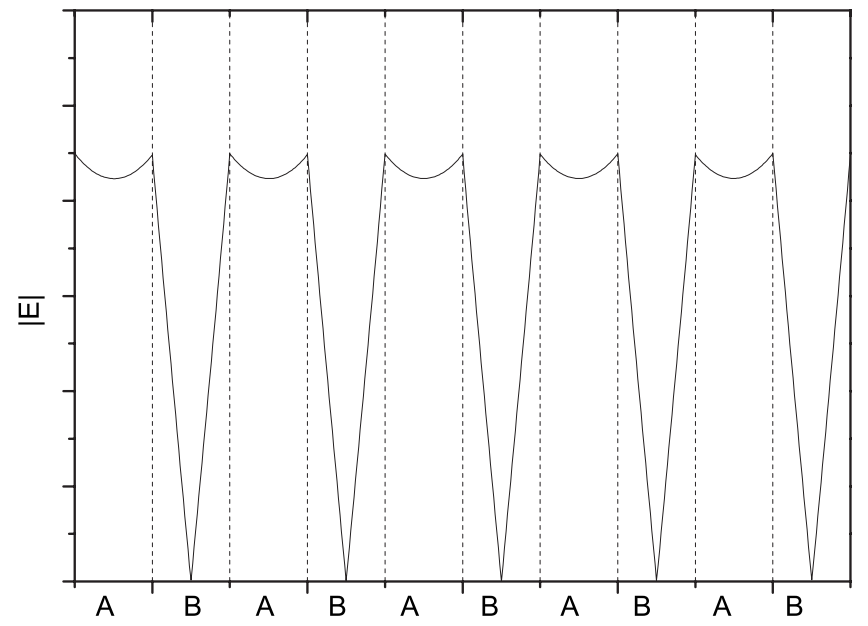

FIG. 1. A typical form of a wave function at band edge in our model.

single unit cell consisting of two adjacent layers of $A$ and $B$. Since $k_{A}$ is imaginary, the electric field exponentially decays near the input surface of layer $A$. The presence of layer $B$ with an impedance different from that of $A$ adds an exponential growth term to the field inside layer $A$, leading to a cusplike wave function near the interface of $A$ and $B$ layers, which peaks at the interface and exponentially decays into the layers, mimicking a surface state. In a periodic structure, this cusplike wave function repeats itself and, therefore, facilitates the wave transport through the sample. A typical form of such wave function at band edge is shown in Fig. 1.

To study the wave localization behavior in random systems, we calculate the transmission coefficient as a function of sample thickness, $T(L)$. For localized waves, $T(L)$ exponentially decays with $L$, apart from some statistical fluctuations. The localization length $\xi$ is defined by ${ }^{13}$

$$
\xi^{-1}=\lim _{L \rightarrow \infty} \frac{-\ln T(L)}{L} .
$$

In the limit of infinite $L$, the above equation gives a welldefined value of $\xi$ through self-averaging. When $L$ is finite, the localization length can be obtained from the geometrical mean of $T$ over many different configurations, i.e., $\xi^{-1}=$ $-\langle\ln T(L)\rangle / L$. Typically, in our calculations, the layer thickness is taken to be five to six times of the localization length and the number of configurations is chosen to be 500, which is found to be sufficient for obtaining a reliable result.

The transmission coefficient of a layered system can be calculated by using the standard transfer-matrix method. ${ }^{6,14,15}$ The field in the $i$ th layer can be described by a vector $u_{i}$ composed of two components: electric $(E)$ and magnetic $(H)$ fields, respectively. The transfer matrix relates the field in the $(i+1)$ th layer to the $i$ th layer through $u_{i+1}$ $=M_{i} u_{i}$, where $M_{i}$ has the form

$$
M_{i}=\left[\begin{array}{cc}
\cosh \left|k_{i}\right| d_{i} & 1 /\left|q_{i}\right| \sinh \left|k_{i}\right| d_{i} \\
-\left|q_{i}\right| \sinh \left|k_{i}\right| d_{i} & \cosh \left|k_{i}\right| d_{i}
\end{array}\right] .
$$

$T$ is obtained by evaluating ${ }^{6}$ 


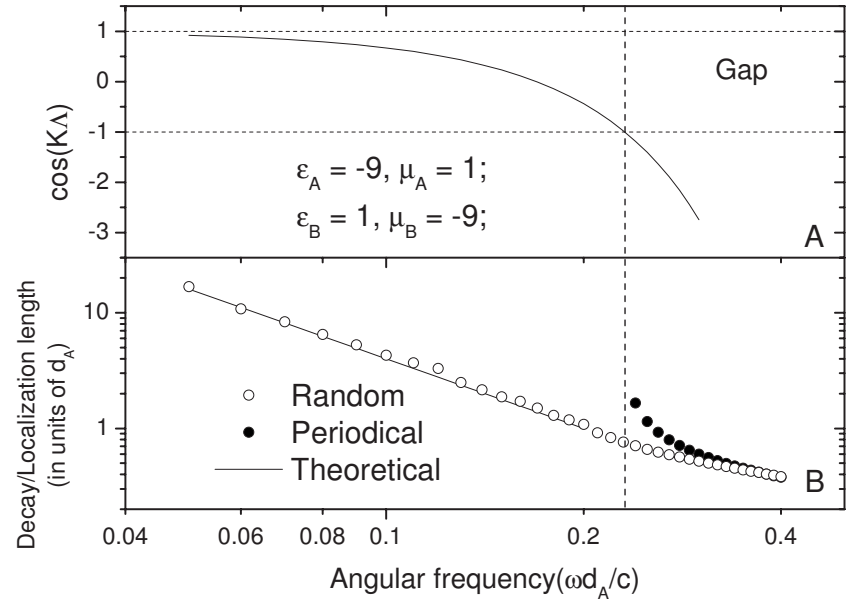

FIG. 2. A nondispersive model. Band structure and localization length as a function of dimensionless angular frequency for the first type of SNG systems described in the text.

$$
T=\left(\frac{2}{m_{11}+m_{22}-m_{12}-m_{21}}\right)^{2},
$$

where $m_{i, j}(i, j=1,2)$ are the matrix elements of $M^{(N)}=\Pi_{i=1}^{N}$ $M_{i}$, and $N$ is the total number of layers.

\section{SIMULATION RESULTS OF A NONDISPERSIVE MODEL}

For simplicity, we first study a nondispersive model, in which $\varepsilon_{A, B}$ and $\mu_{A, B}$ are independent of frequency. The band structures of a periodical structure, i.e., $(A B)^{n}$, can be obtained by using Eq. (2). For the nondispersive model, we find, in general, two types of band structures. The first type is shown in Fig. 2(a), where we have chosen $\varepsilon_{A}=-9, \mu_{A}=1$, $\varepsilon_{B}=1, \mu_{B}=-9$, and $d_{A}=d_{B}$. In Fig. 2(a), we have plotted $\cos (K \Lambda)$ versus the logarithm of the dimensionless angular frequency $\widetilde{\omega} \equiv \omega d_{A} / c$, where $c$ is the wave speed in vacuum. The second type is shown in Fig. 3(a), where we have chosen

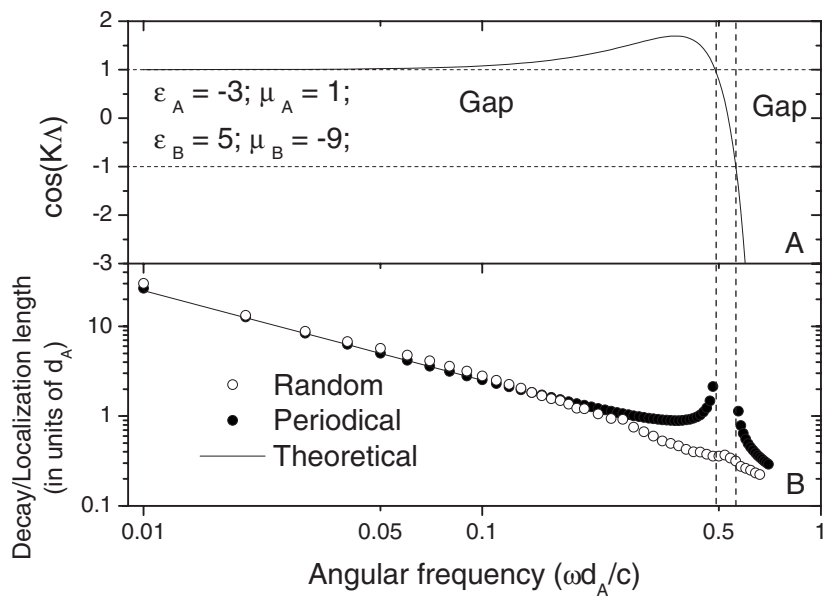

FIG. 3. A nondispersive model. Band structure and localization length as a function of dimensionless angular frequency for the second type of SNG systems described in the text.
$\varepsilon_{A}=-3, \mu_{A}=1, \varepsilon_{B}=5$, and $\mu_{B}=-9$. These two types can be characterized by their wave propagation properties at low frequencies, i.e., propagating in Fig. 2(a), while nonpropagating in Fig. 3(a). The propagation properties at long wavelengths can be determined by the signs of two effective parameters: effective permittivity, $\varepsilon_{e f f}=\frac{\varepsilon_{A} d_{A}+\varepsilon_{B} d_{B}}{d_{A}+d_{B}}$, and effective permeability, $\mu_{e f f}=\frac{\mu_{A} d_{A}+\mu_{B} d_{B}}{d_{A}+d_{B}}$. Here, we have applied the quasistatic effective medium theory for scalar waves to both electric and magnetic fields as they both are scalar waves in a $1 \mathrm{D}$ system with normal incidence. ${ }^{16}$ For the first type as shown in Fig. 2(a), both $\varepsilon_{e f f}=-4$ and $\mu_{e f f}=-4$ are negative, leading to a propagating band with a negative refractive index at low frequencies. For the second type, either $\varepsilon_{\text {eff }}$ or $\mu_{\text {eff }}$ is negative, i.e., their signs are opposite. In this case, the band structures show a gap at low frequencies as shown in Fig. 3(a), where $\varepsilon_{e f f}=1$ and $\mu_{e f f}=-4$. Such an effective medium description is valid only when the wavelength in the effective medium is much larger than the period, i.e., $2 \pi c / \omega \sqrt{\left|\varepsilon_{e f f} \mu_{e f f}\right|} \gg d_{A}+d_{B}$. At higher frequencies, for the first type, it is seen from Fig. 2(a) that a transition to a gap occurs at a particular frequency at which $\cos (K \Lambda)=-1$, whereas for the second type, the band structures show two gaps, sandwiching a pass band as shown in Fig. 3(a).

When the above systems are random, we are interested in studying their localization behaviors. For this purpose, we consider a finite system with any given number of periods. To introduce the randomness, we rearrange $A$ and $B$ layers in a random manner as mentioned in Sec. II. By using the transfer-matrix method, we calculate the transmission coefficient $T$ for each random configuration. For any chosen frequency and sample thickness $L, T$ is calculated for 500 different configurations to study the localization behavior. A localized state is identified when the configurational average of $-\ln T$ is proportional to $L$, and the localization length is obtained through $\xi=-L /\langle\ln T\rangle$. For both systems shown in Figs. 2(a) and 3(a), we find that all states are localized. The corresponding results of the localization length are shown in Figs. 2(b) and 3(b) by open circles. Although the localization of waves for 1D systems is well known, there are some interesting differences between the random SNG and DP systems.

In Figs. 2(b) and 3(b), we also plot the decay length of the corresponding periodical systems by solid circles for frequencies inside the gap. Here, we define the decay length as $\xi_{d}=-L / \ln T$. It is interesting to see that within the gap, the localization length is, in general, smaller than the decay length, except in a region near $\omega d_{A} / c=0.05$ in Fig. 3(b), where the localization length is slightly larger than the decay length. This is different from the situation found in traditional random DP systems. In Fig. 4, we show the localization length and the decay length of a typical DP system for comparison. It is clearly seen that the localization length inside a gap is, in general, much larger than the decay length, except near the band edge. For DP systems, this result is normally explained as follows. The randomness introduces new states inside a gap and, therefore, facilitates the transport of waves, leading to a larger transmission. Or alternatively, the formation of a gap in a periodic DP system is a result of complete destructive interferences among various 


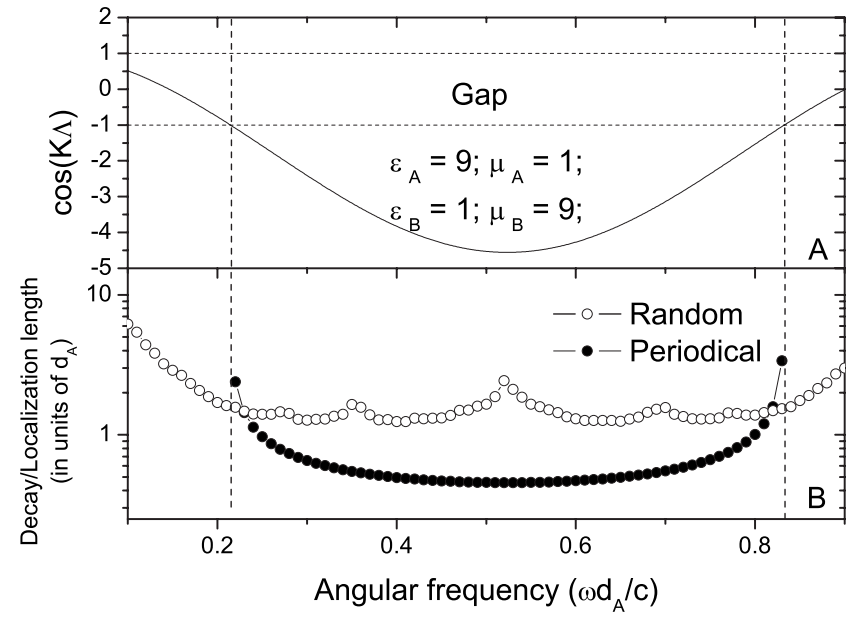

FIG. 4. Band structure and localization length as a function of dimensionless angular frequency for double-positive systems.

partial waves in the forward direction. The introduction of randomness will reduce such interferences and make them incomplete, leading to a larger transmission. Thus, the localization length is generally larger than the decay length of the corresponding periodical structures. However, in SNG materials, the wave is intrinsically evanescent in each layer. The transport of waves is facilitated by the presence of a cusplike wave function near the interface of $A$ and $B$ layers due to the coupling of evanescent waves in each layer as discussed in Sec. II. So the number of interfaces in the systems plays an important role for the wave propagation in SNG systems. In the periodical system $(A B)^{n}, A$ and $B$ layers are alternatively and periodically stacked. However, in our random model, $A$ and $B$ layers are randomly stacked. Thus, the total number of the interfaces between $A$ and $B$ layers is always less than that in a periodic sample. This can make the localization length in a random sample smaller than the decay length of the corresponding periodical sample.

Figures 2(b) and 3(b) also exhibit different localization behaviors at low frequencies, i.e., $\xi \propto \widetilde{\omega}^{-2}$ in Fig. 2(b) and $\xi$ $\propto \widetilde{\omega}^{-1}$ in Fig. 3(b). The difference can also be understood from the different long-wavelength limit properties of two random systems. In the long-wavelength limit, the periodic systems can be analyzed by using effective medium theory as discussed above. For the first type of system shown in Fig. 2 , the underlying periodic system can be considered as a homogeneous medium with $\varepsilon_{e f f}=-4$ and $\mu_{e f f}=-4$. This belongs to the so-called left-handed materials, where waves can propagate with a negative refractive index. Since this is a propagating band for the periodic system, the localization length behavior in the random system can be theoretically analyzed by using the following formula derived for 1D random DP systems: ${ }^{17}$

$$
\begin{aligned}
\xi^{-1}= & \frac{1}{4}\left[\frac{d_{A}^{2}}{d_{A}+d_{B}}\left(\left|\hat{\varepsilon}_{A}\right|+\left|\widehat{\mu_{A}}\right|\right)^{2}+\frac{d_{B}^{2}}{d_{A}+d_{B}}\left(\left|\hat{\varepsilon}_{B}\right|\right.\right. \\
& \left.\left.+\left|\widehat{\mu_{B}}\right|\right)^{2}\right] \varepsilon_{m} \mu_{m} \omega^{2} .
\end{aligned}
$$

The detailed derivation and the definition of the parameters
$\hat{\varepsilon}_{A(B)}, \hat{\mu}_{A(B)}, \varepsilon_{m}$, and $\mu_{m}$ are given in the Appendix. It should be pointed out that the expressions of the averaged parameters $\varepsilon_{m}$ and $\mu_{m}$, i.e., $\varepsilon_{m}=\frac{\varepsilon_{A} d_{A}+\varepsilon_{B} d_{B}}{d_{A}+d_{B}}$ and $\mu_{m}=\frac{\mu_{A} d_{A}+\mu_{B} d_{B}}{d_{A}+d_{B}}$, are exactly the same as those of $\varepsilon_{\text {eff }}$ and $\mu_{\text {eff }}$ defined before for an ordered metamaterial. Here, we purposely choose a different subscript because the concepts of homogenization and, therefore, effective medium are not appropriate for 1D random media due to wave localization. Equation (6) gives $\xi / d_{A}=0.04 \widetilde{\omega}^{-2}$, which is plotted by a solid line in Fig. 2(b). It is clearly seen that the theoretical result agrees well with the stimulation result. It should be pointed out again that the above theory works only when the long-wavelength limit description is valid. For the system considered in Fig. 2, it requires that $\widetilde{\omega} \equiv \omega d_{A} / c \ll \pi / 4$. This condition is satisfied for the range of $\widetilde{\omega}$ shown in Fig. 2. For the second type shown in Fig. 3, it is found that the localization length coincides with the decay length in the long-wavelength limit. In this limit, the waves are effectively evanescent with the decay length given by $c /\left(2 \sqrt{\left|\varepsilon_{\text {eff }} \mu_{\text {eff }}\right|} \omega\right)$ or $\xi_{d} / d_{A}=0.25 \widetilde{\omega}^{-1}$. This result is plotted by a solid curve in Fig. 3(b), which again shows excellent agreement with the simulation result when $\widetilde{\omega}$ $<0.2$.

\section{SIMULATION RESULTS OF A DISPERSIVE MODEL}

Generally, the metamaterials are dispersive, i.e., $\varepsilon$ and $\mu$ are frequency dependent. One unique feature of a dispersive model is the existence of a gap near a particular frequency at which the sum of the two impedances of the $A$ and $B$ layers vanishes. This special gap is called SNG gap in Ref. 6. Here, we are interested in the localization behavior inside a SNG gap. For convenience, we use the following model ${ }^{6,18}$ dispersion to describe the isotropic single-negative materials:

$$
\begin{aligned}
& \varepsilon_{A}=a, \quad \mu_{A}=1-\frac{\omega_{c}^{2}}{\omega^{2}}, \\
& \varepsilon_{B}=1-\frac{\omega_{p}^{2}}{\omega^{2}}, \quad \mu_{B}=b,
\end{aligned}
$$

where $\omega_{p}$ and $\omega_{c}$ are, respectively, the electronic and magnetic resonance frequencies, and $a$ and $b$ are positive constants. In this model, $\varepsilon_{A}$ and $\mu_{B}$ are fixed to be positive, while $\mu_{A}$ and $\varepsilon_{B}$ are frequency dependent. In the following calculations, we choose $a=3, b=1, \omega_{p}^{2}=0.16\left(c / d_{0}\right)^{2}$, and $\omega_{c}^{2}=0.08\left(c / d_{0}\right)^{2}$, where $d_{0}$ denotes the characteristic length of the metamaterials. It should be noted that all metameterials possess absorption. This will introduce an imaginary part into the terms $\mu_{A}$ and $\varepsilon_{B}$ in Eq. (7). This, in turn, will give rise to a different decay mechanism to the electromagnetic field. Since the interplay between absorption and localization can mask the pure localization behavior we want to investigate, in this work, we do not include absorption in Eq. (7). In Fig. 5(a), we plot $\mu_{A}$ and $\varepsilon_{B}$ as functions of dimensionless angular frequency $\hat{\omega} \equiv \omega d_{0} / c$. The SNG metamaterials refer to the region of $\hat{\omega}^{2}<0.08$ or $\hat{\omega}<0.2828$, in which both $\mu_{A}$ and $\varepsilon_{B}$ are negative as can be directly seen from Eq. (7).

By using Eq. (2), we calculate the band structures of the 1D periodical systems made of these kinds of materials. The 


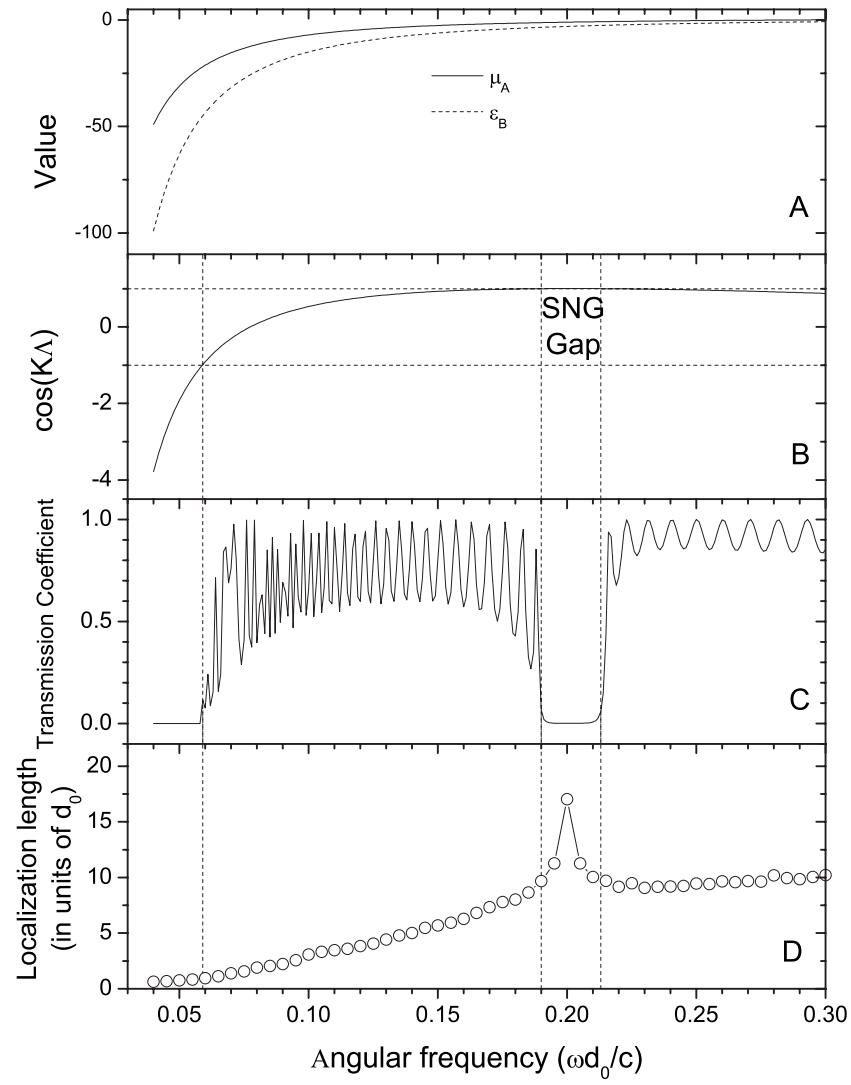

FIG. 5. A dispersive model for SNG systems. (A) Dispersion relations of $\varepsilon$ and $\mu,(\mathrm{B})$ band structure, $(\mathrm{C})$ transmission coefficient of a periodic system, and (D) localization length of random systems.

result of the case $d_{B}=1.2 d_{0}$ and $d_{A}=d_{0}$ is shown in Fig. 5(b). The corresponding transmission spectrum is shown in Fig. $5(\mathrm{c})$. It can be seen that within the $\mathrm{SNG}$ region, i.e., $\hat{\omega}$ $<0.2828$, there exist both pass bands and stop bands. The interesting SNG gap found in Ref. 6 is located around $\hat{\omega}_{0}$ $=0.2$, at which $q_{A}+q_{B}=0 .{ }^{19}$ The existence of this SNG gap can be seen from Eq. (2). At $\hat{\omega}_{0}$, the equation can be reduced to $\cosh \left(\left|k_{A}\right| d_{A}-\left|k_{B}\right| d_{B}\right)$, which is greater or equal to 1 . Only when $\left|k_{A}\right| d_{A}=\left|k_{B}\right| d_{B}$, we have $\cosh \left(\left|k_{A}\right| d_{A}-\left|k_{B}\right| d_{B}\right)=1$, which corresponds to a tunneling or transparency. ${ }^{5,20}$ However, in the most general situation, $\left|k_{A}\right| d_{A} \neq\left|k_{B}\right| d_{B}$ and $\cosh \left(\left|k_{A}\right| d_{A}\right.$ $\left.-\left|k_{B}\right| d_{B}\right)>1$, this leads to a gap around $\hat{\omega}_{0}$. The formation mechanism of the SNG gap is different from that of the Bragg gap in DP systems. It is also unique to the dispersive model considered here and is different from the gaps found in Figs. 2(a) and 3(a) for the nondispersive models. This unique formation mechanism of the SNG gap is also the source of various anomalous localization properties, which will be discussed later, when randomness is introduced into the system.

When randomness is introduced into the system, we calculate the localization length and plot the result in Fig. 5(d). It is surprising to see that the localization length has a sharp peak at $\hat{\omega}_{0}=0.2$ inside the SNG gap. Such a sharp peak is absent in random DP systems such as the one shown in Fig. 4. In order to find the origin of this anomalous peak, we also calculate the decay length of the corresponding periodical

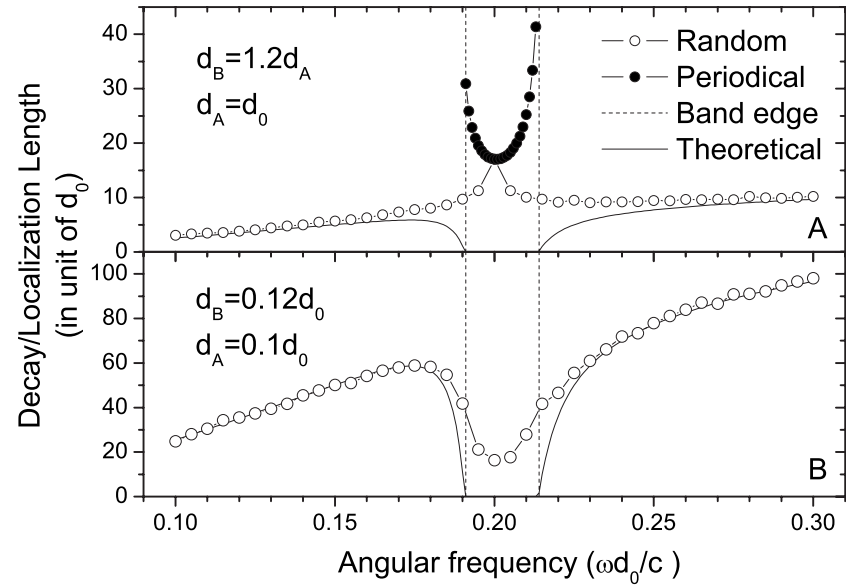

FIG. 6. (A) The open circles and solid curves are the simulation and theoretical results, respectively, of the localization length of random SNG systems. The solid circles denote the decay length of the corresponding periodical system. The vertical lines show the position of the SNG gap. (B) Same as (A), except that the layer thickness of each layer is reduced by a factor of 10 .

systems. The result is plotted by solid circles in Fig. 6, where the open circles denote the localization length and the two dashed lines mark the gap region. The first thing we noticed is that the localization length $\xi$ is always smaller than the decay length $\xi_{d}$ except at $\hat{\omega}_{0}$, where two lengths become equal. The reason that $\xi=\xi_{d}$ at $\hat{\omega}_{0}$ can be understood from the transfer matrix $M_{i}$ given in Eq. (3), which can be rewritten as

$$
M_{i}=\left(\begin{array}{cc}
1 & 1 \\
q_{i} & -q_{i}
\end{array}\right)\left(\begin{array}{cc}
e^{-\left|k_{i}\right| d_{i}} & 0 \\
0 & e^{\left|k_{i}\right| d_{i}}
\end{array}\right)\left(\begin{array}{cc}
1 & 1 \\
q_{i} & -q_{i}
\end{array}\right)^{-1} .
$$

By using the above expression, it is easy to show that, when $q_{A}+q_{B}=0, M_{A}$ and $M_{B}$ become commutable and have the following form:

$$
\begin{aligned}
M_{A} M_{B}= & \left(\begin{array}{cc}
1 & 1 \\
q_{A} & -q_{A}
\end{array}\right)\left(\begin{array}{cc}
0 & e^{-\left(\left|k_{A}\right| d_{A}-\left|k_{B}\right| d_{B}\right)} \\
e^{\left(\left|k_{A}\right| d_{A}-\left|k_{B}\right| d_{B}\right)} & 0
\end{array}\right) \\
& \times\left(\begin{array}{cc}
1 & 1 \\
q_{B} & -q_{B}
\end{array}\right)^{-1},
\end{aligned}
$$

where $k_{A}$ and $k_{B}$ are wave vectors at $\hat{\omega}_{0}$ for the $A$ and $B$ layers, respectively. Thus, the transmission coefficient of any random configuration is the same as that of a periodic structure, leading to $\xi=\xi_{d}$. Furthermore, it can also be shown that a product of $4 N$ matrices of the form $\left(M_{A} M_{B} M_{B} M_{A}\right)^{N}$ has the following simple expression:

$$
\begin{aligned}
\left(M_{A} M_{B} M_{B} M_{A}\right)^{N}= & \left(\begin{array}{cc}
1 & 1 \\
q_{A} & -q_{A}
\end{array}\right) \\
& \times\left(\begin{array}{cc}
e^{-2 N\left(\left|k_{A}\right| d_{A}-\left|k_{B}\right| d_{B}\right)} & 0 \\
0 & e^{2 N\left(\left|k_{A}\right| d_{A}-\left|k_{B}\right| d_{B}\right)}
\end{array}\right) \\
& \times\left(\begin{array}{cc}
1 & 1 \\
q_{A} & -q_{A}
\end{array}\right)^{-1}
\end{aligned}
$$

from which we can obtain the decay length $\xi_{d}=0.5\left(d_{A}\right.$ $\left.+d_{B}\right) /|| k_{A}\left|d_{A}-\right| k_{B}\left|d_{B}\right|$. For the dispersive model discussed 


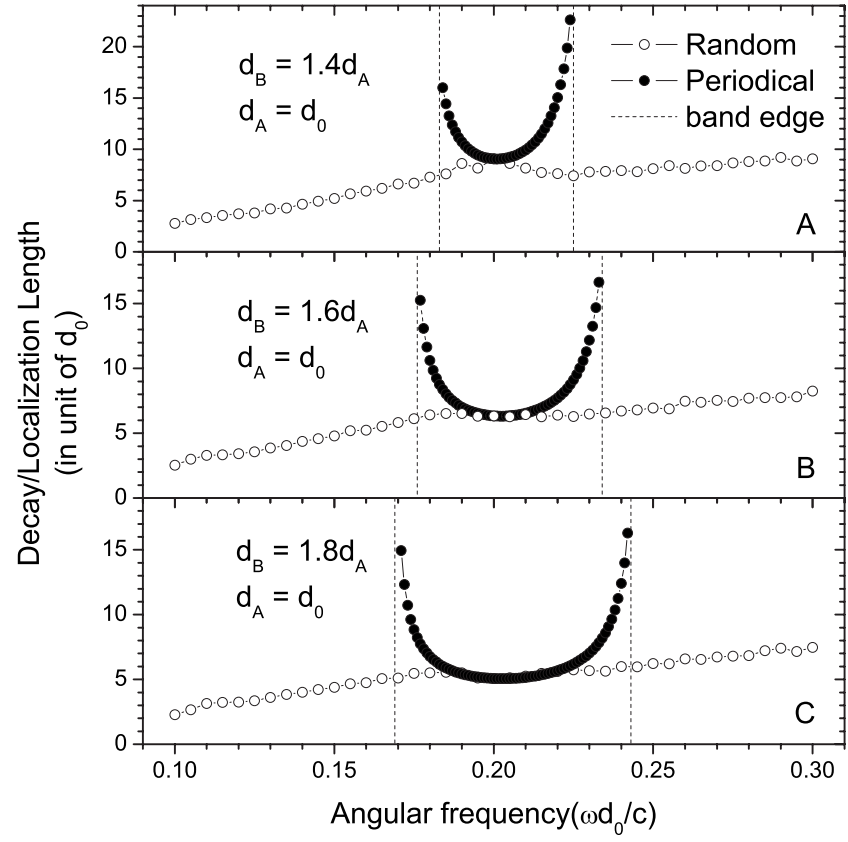

FIG. 7. The localization length and the decay length in SNG systems with three different ratios of $d_{A} / d_{B}$.

above, the above relation gives $\xi_{d} / d_{0}=1.4433\left(d_{A}+d_{B}\right) / \mid d_{A}$ $-d_{B} \mid$. Thus, $\xi_{d}$ diverges when $d_{A}=d_{B}$, at which the gap is closed. Thus, the sharp peak appears in the SNG gap is a unique property associated with the special frequency $\hat{\omega}_{0}$ at which $q_{A}+q_{B}=0$. To numerically verify this, we have done similar calculations by choosing $d_{B} / d_{A}=1.4,1.6$, and 1.8 and $d_{A}=d_{0}$. These results are plotted in Fig. 7. It is clearly seen that the height of the peak at $\hat{\omega}_{0}$ continuously decreases and at the same time, the width of the gap increases. The peak disappears when $d_{B} / d_{A} \geqslant 1.6$. At the same time, the difference between $\xi$ and $\xi_{d}$ decreases significantly in a region centered at $\hat{\omega}_{0}$. In Fig. 8, we plot the localization length at $\hat{\omega}_{0}$ as a function of $d_{B}$ for two different choices of $d_{A}$ : one with

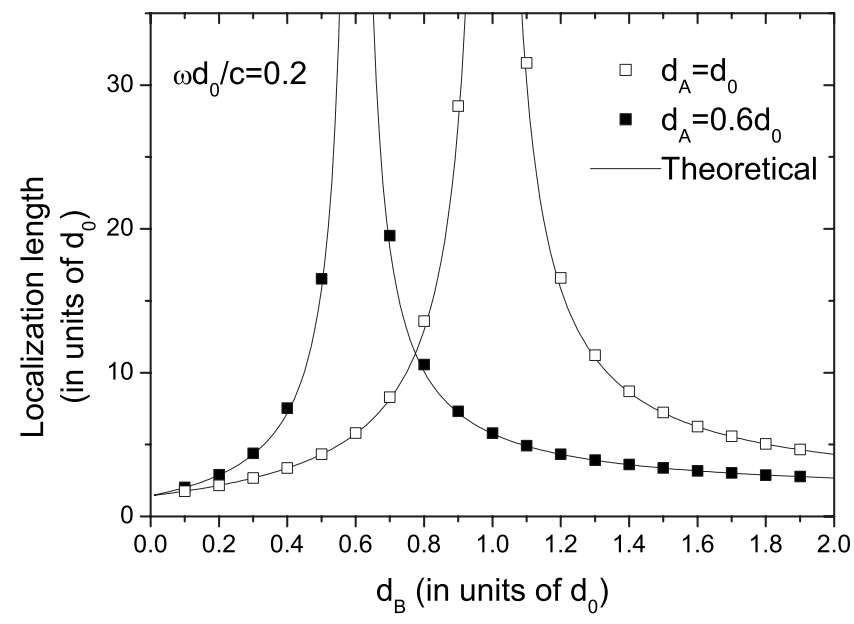

FIG. 8. The simulated localization length at $\omega d_{0} / c=0.2$ as a function of $d_{B}$ for $d_{A}=d_{0}$ (open squares) and $d_{A}=0.6 d_{0}$ (solid squares). The solid curves are the analytical results obtained form transfer matrices.

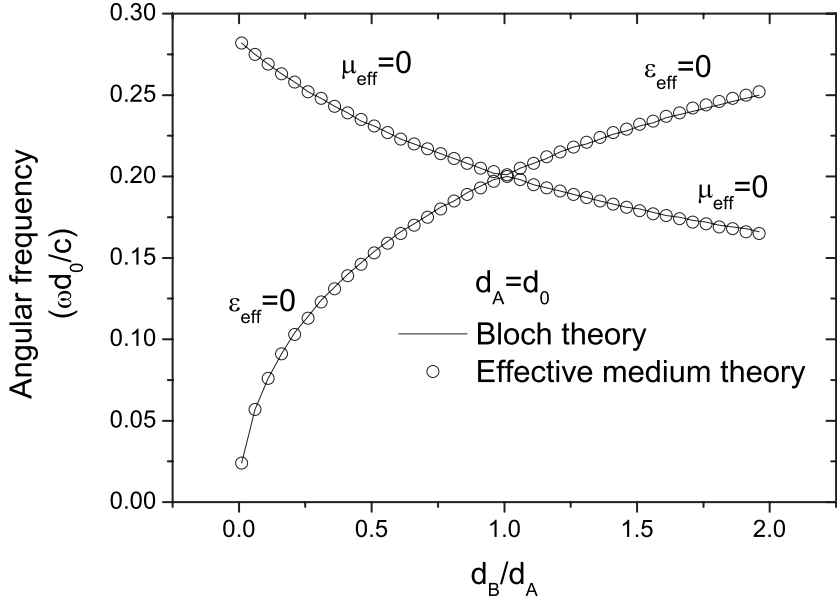

FIG. 9. The position of the SNG gap as a function of $d_{B} / d_{A}$ for $d_{A}=d_{0}$. The solid curves and open circles represent, respectively, the results of Eq. (1) and the effective medium theory.

$d_{A}=d_{0}$ and the other with $d_{A}=0.6 d_{0}$. It is clearly seen that both curves follow exactly the relation $\xi_{d} / d_{0}=1.4433\left(d_{A}\right.$ $\left.+d_{B}\right) /\left|d_{A}-d_{B}\right|$, which is shown by the solid curves.

In order to quantitatively understand how the gap size changes with the ratio $d_{B} / d_{A}$, we plot in Fig. 9 the frequencies of the two band edges as functions of $d_{B} / d_{A}$ from 0 to 2 by two solid curves. These two curves are obtained from Eq. (2) when $\cos K \Lambda=1$. For frequencies inside the gap, we find $\cos K \Lambda>1$. To understand these two curves, one can use the effective medium approach. The open circles in Fig. 9 are the results obtained from the relations $\varepsilon_{\text {eff }}=\frac{\varepsilon_{A} d_{A}+\varepsilon_{B} d_{B}}{d_{A}+d_{B}}=0$ and $\mu_{\text {eff }}=\frac{\mu_{A} d_{A}+\mu_{B} d_{B}}{d_{A}+d_{B}}=0$. The excellent agreement between the open circles and the solid curves indicate that the region between these two band edges effectively represents an evanescent region of the effective medium, i.e., $\varepsilon_{\text {eff }} \mu_{\text {eff }}<0$. In fact, by using the condition $\cos K \Lambda=1$ together with the long-wavelength conditions that $\left|k_{A}\right| d_{A} \ll 1$ and $\left|k_{B}\right| d_{B} \ll 1$, we are able to show that Eq. (2) reduces to $\varepsilon_{\text {eff }}=0$ or $\mu_{\text {eff }}=0$. Thus, the position of the SNG gap can be well described by the effective medium description.

Finally, we discuss the localization behavior outside the SNG gap. We calculate the localization length by using Eq. (6) and plot it by two solid curves in Fig. 6. It should be mentioned that Eq. (6) is derived for random DP materials. For SNG materials, this equation can only be applied to the propagating regions. It is interesting to see that Eq. (6) can well reproduce the simulation result if frequency is not close to the two band edges of the SNG gap. As the band edge is approached, the theory predicts a decreasing localization length which vanishes at the band edge, whereas the simulation result shows an increasing localization length. The discrepancy between the two can be understood as follows. Near the band edges, either $\varepsilon_{m}$ or $\mu_{m}$ is very small. Thus, the normalized fluctuations defined by Eq. (A1) of the Appendix become very large, which implies a strongly disordered system. In this case, the long-wavelength theory derived in the Appendix becomes inadequate. Better agreement can be achieved by reducing the layer thicknesses $d_{A}$ and $d_{B}$. This is demonstrated in Fig. 6(b), where we have chosen $d_{A}=0.1 d_{0}$ 
and $d_{B}=0.12 d_{0}$. The open circles in the figure represent the simulation result, and the solid curve is the result of Eq. (6). Compared to the case of Fig. 6, since both $d_{A}$ and $d_{B}$ are smaller by a factor of 10 , the localization length in the propagating band is increased by the same factor as can be seen from Eq. (6). However, inside the SNG gap, the localization length (or decay length) at $\hat{\omega}_{0}$ remains unchanged as it depends only on the ratio $d_{A} / d_{B}$ as can be seen from the relation $\xi_{d} / d_{0}=1.4433\left(d_{A}+d_{B}\right) /\left|d_{A}-d_{B}\right|$. Thus, the localization length changes from an increasing function to a decreasing function as the band edge is approached, leading to a better agreement with the prediction of Eq. (6).

\section{CONCLUSION}

We have investigated the Anderson localization behavior in one-dimensional random systems made of SNG materials. Both the nondispersive and dispersive cases are studied. We find that the localization behavior in the propagating regions is similar to that of the traditional random systems made of DP materials. For the dispersive model, our interest is focused on a special gap created around a particular frequency at which the sum of the two impedances of the SNG materials vanishes. Some other localization behaviors have been found inside this gap. Analytical methods based on transfer matrices and long-wavelength limit description have been used to explain various transport properties associated with this gap.

\section{ACKNOWLEDGMENTS}

This work was supported by the Hong Kong RGC Grant No. HKUST3/06C. Computational resources were supported by the Shun Hing Education and Charity Fund. P.H. also acknowledges support by the National Natural Science Foundation of China under Grant No. 10504008.

\section{APPENDIX}

The derivation of Eq. (6) is based on a standard analytical result developed for classical waves in the long-wavelength limit. ${ }^{17}$ In order to use the result for the two-component random systems we study here, we rewrite the permittivity $\varepsilon$ and permeability $\mu$ of each component in terms of their mean values, i.e.,

$$
\varepsilon=\varepsilon_{m}(1+\hat{\varepsilon},)
$$

and

$$
\mu=\mu_{m}(1+\hat{\mu}),
$$

where $\varepsilon_{m}=\frac{\varepsilon_{A} d_{A}+\varepsilon_{B} d_{B}}{d_{A}+d_{B}}$ and $\mu_{m}=\frac{\mu_{A} d_{A}+\mu_{B} d_{B}}{d_{A}+d_{B}}$. It should be noted that although the expressions of $\varepsilon_{m}$ and $\mu_{m}$ are exactly the same as those of $\varepsilon_{\text {eff }}$ and $\mu_{\text {eff }}$ defined in Sec. III, we choose a different subscript here to avoid possible confusion as the concept of homogenization and effective medium no longer holds in 1D random media due to wave localization. $\hat{\varepsilon}$ and $\hat{\mu}$ denote the normalized fluctuation in $\varepsilon$ and $\mu$ as follows:

$$
\hat{\varepsilon}=\frac{\varepsilon-\varepsilon_{m}}{\varepsilon_{m}}
$$

and

$$
\hat{\mu}=\frac{\mu-\mu_{m}}{\mu_{m}},
$$

where $\varepsilon(\mu)$ can be $\varepsilon_{A}$ or $\varepsilon_{B}\left(\mu_{A}\right.$ or $\left.\mu_{B}\right)$ with probability $P_{A}=d_{A} /\left(d_{A}+d_{B}\right)$ and $P_{B}=d_{B} /\left(d_{A}+d_{B}\right)$, respectively.

According to the work of Sheng et al., ${ }^{17}$ the localization length $\xi$ has the following simple expression in the longwavelength limit:

$$
\xi^{-1}=\frac{1}{2}\left(\alpha_{\varepsilon \varepsilon}+\alpha_{\mu \mu}-2 \alpha_{\varepsilon \mu}\right) \varepsilon_{m} \mu_{m} \omega^{2}
$$

where

$$
\begin{aligned}
\alpha_{\varepsilon \varepsilon} & =\int_{0}^{\infty}\langle\hat{\varepsilon}(0) \hat{\varepsilon}(x)\rangle d x, \\
\alpha_{\mu \mu} & =\int_{0}^{\infty}\langle\hat{\mu}(0) \hat{\mu}(x)\rangle d x,
\end{aligned}
$$

and

$$
\alpha_{\varepsilon \mu}=\int_{0}^{\infty}\langle\hat{\varepsilon}(0) \hat{\mu}(x)\rangle d x .
$$

It should be pointed out that the above expression for the localization length is valid as long as $\varepsilon_{m} \mu_{m}>0$. Thus, it is applicable to our random systems when both $\varepsilon_{m}<0$ and $\mu_{m}<0$. The above $\alpha$ 's are integrals of the autocorrelation and correlation functions for $\hat{\varepsilon}$ and $\hat{\mu}$. Since there are no correlations between two layers, $\langle\hat{\varepsilon}(0) \hat{\varepsilon}(x)\rangle \neq 0$ only when $\hat{\varepsilon}(0)$ and $\hat{\varepsilon}(x)$ lie in the same layer, yielding

$$
\alpha_{\varepsilon \varepsilon}=P_{A} \int_{0}^{d_{A}}\left(1-\frac{x}{d_{A}}\right)\left(\hat{\varepsilon}_{A}\right)^{2} d x+P_{B} \int_{0}^{d_{B}}\left(1-\frac{x}{d_{B}}\right)\left(\hat{\varepsilon}_{B}\right)^{2} d x
$$

or,

$$
\alpha_{\varepsilon \varepsilon}=\frac{1}{2}\left[P_{A} d_{A}\left(\hat{\varepsilon}_{A}\right)^{2}+P_{B} d_{B}\left(\hat{\varepsilon}_{B}\right)^{2}\right]
$$

In the above equation, $\left(1-\frac{x}{d_{A(B)}}\right)$ stands for the probability that two points separated by $x$ can still lie in the same layer, and the two terms on the right-hand side of the above equation stand for the two points that lie in the $A$ layers and $B$ layers, respectively. Similarly, we have $\alpha_{\mu \mu}=\frac{1}{2}\left[P_{A} d_{A}\left(\hat{\mu}_{A}\right)^{2}\right.$ $\left.+P_{B} d_{B}\left(\hat{\mu}_{B}\right)^{2}\right]$ and $\alpha_{\varepsilon \mu}=\frac{1}{2}\left(P_{A} d_{A} \hat{\varepsilon}_{A} \hat{\mu}_{A}+P_{B} d_{B} \hat{\varepsilon}_{B} \hat{\mu}_{B}\right)$. By substituting the above expressions of $\alpha_{\varepsilon \varepsilon}, \alpha_{\mu \mu}$, and $\alpha_{\varepsilon \mu}$ into Eq. (A3), we have

$$
\xi^{-1}=\frac{1}{4}\left[P_{A} d_{A}\left(\left|\hat{\varepsilon}_{A}\right|+\left|\widehat{\mu_{A}}\right|\right)^{2}+P_{B} d_{B}\left(\left|\hat{\varepsilon}_{B}\right|+\left|\widehat{\mu_{B}}\right|\right)^{2}\right] \varepsilon_{m} \mu_{m} \omega^{2} .
$$


${ }^{1}$ J. B. Pendry, A. J. Holden, D. J. Robbins, and W. J. Stewart, IEEE Trans. Microwave Theory Tech. 47, 2075 (1999).

${ }^{2}$ D. R. Smith, W. J. Padilla, D. C. Vier, S. C. Nemat-Nasser, and S. Schultz, Phys. Rev. Lett. 84, 4184 (2000); R. A. Shelby, D. R. Smith, and S. Schultz, Science 292, 77 (2001).

${ }^{3}$ J. B. Pendry, Phys. Rev. Lett. 85, 3966 (2000).

${ }^{4}$ J. Li, L. Zhou, C. T. Chan, and P. Sheng, Phys. Rev. Lett. 90, 083901 (2003).

${ }^{5}$ H. Jiang, H. Chen, H. Li, Y. Zhang, J. Zi, and S. Y. Zhu, Phys. Rev. E 69, 066607 (2004).

${ }^{6}$ L. G. Wang, H. Chen, and S. Y. Zhu, Phys. Rev. B 70, 245102 (2004).

${ }^{7}$ I. V. Shadrivov, A. A. Sukhorukov, and Y. S. Kivshar, Phys. Rev. Lett. 95, 193903 (2005).

${ }^{8}$ P. W. Anderson, Phys. Rev. 109, 1492 (1958).

${ }^{9}$ Scattering and Localization of Classical Waves in Random Media, edited by P. Sheng (World Scientific, Singapore, 1990).

${ }^{10}$ Y. Dong and X. Zhang, Phys. Lett. A 359, 542 (2006).

${ }^{11}$ A. A. Asatryan, L. C. Botten, M. A. Byrne, V. D. Freilikher, S. A. Gredeskul, I. V. Shadrivov, R. C. McPhedran, and Y. S. Kivshar, Phys. Rev. Lett. 99, 193902 (2007).

${ }^{12}$ A. Yariv and P. Yeh, Optical Waves in Crystals, Propagation and
Control of Laser Radiation (Wiley, New York, 1984).

${ }^{13}$ I. M. Lifshits, S. A. Gredeskul, and L. A. Pastur, Introduction to the Theory of Disordered Systems (Wiley, New York, 1988).

${ }^{14} \mathrm{M}$. Born and E. Wolf, Principles of Optics, 7th ed. (University of Cambridge Press, Cambridge, 1999).

${ }^{15}$ Z. Knittl, Optics of Thin Films (Wiley, New York, 1976).

${ }^{16}$ In a 1D layered medium with normal incidence, the Maxwell equations become $d H(z) / d z=(i \omega / c) D(z)$ and $d E(z) / d z$ $=(i \omega / c) B(z)$, where $D(z)=\epsilon(z) E(z)$ and $B(z)=\mu(x) H(z)$. Since both the average fields $\langle D(z)\rangle$ and $\langle B(z)\rangle$ are slowly varied functions of $z$ in the long-wavelength limit, we have $\epsilon_{e f f}=\langle\epsilon(z)\rangle$ and $\mu_{\text {eff }}=\langle\mu(z)\rangle$.

${ }^{17}$ P. Sheng, B. White, and Z. Q. Zhang, Scattering and Localization of Classical Waves in Random Media, (Ref. 9), p. 563.

${ }^{18}$ D. R. Fredkin and A. Ron, Appl. Phys. Lett. 81, 1753 (2002).

${ }^{19}$ Please note that the impedances $q=\sqrt{\mu} / \sqrt{\varepsilon}$. For layer A, $\varepsilon_{A}>0$ and $\mu_{A}<0$, then $q_{A}=\frac{i \sqrt{\left|\mu_{A}\right|}}{\sqrt{\varepsilon_{A}}}$. For layer B, $\varepsilon_{B}<0$ and $\mu_{B}>0$, then $q_{B}=\frac{\sqrt{\left|\mu_{B}\right|}}{i \sqrt{\varepsilon_{B}}}$. So $q_{A}$ and $q_{B}$ are purely imaginary and have opposite signs.

${ }^{20}$ A. Alu and N. Engheta, IEEE Trans. Antennas Propag. 51, 2558 (2003). 